\title{
Continuation of acid suppression therapy on intensive care discharge: a clinical audit
}

\author{
Authors: Louise Rayner ${ }^{1}$ and Eve Merry ${ }^{2}$
}

\section{Aims}

It is standard practice in intensive care to commence patients at risk of stress-related mucosal disease (SRMD) on acid suppression therapy (AST). ${ }^{1}$ The aim of this audit was to determine the proportion of patients inappropriately continued on AST at the point of critical care discharge, and to develop a robust intervention to prevent this.

\section{Methods}

Audit cycle one

Data were collected from all patients discharged from St George's Hospital general intensive care unit (GICU) over a 14-day period who received AST during their ICU admission. Indications for continuation of AST were taken from local and national guidelines. ${ }^{2,3}$

Of 44 discharged patients, $32 \%$ (14 patients) were continued on AST inappropriately. Of these, 12 patients no longer had a valid indication for AST and two were on the wrong AST.

\section{Intervention}

1 An oral presentation to the GICU MDT of audit findings and local and national guidelines regarding indications for AST.

2 Addition of a prompt to the GICU discharge summary to encourage review of AST at the point of discharge to the ward.

\section{Results}

\section{Audit cycle two}

Data were collected, as above, 1 month following the intervention. Of 27 discharged patients, 15\% (three patients) were inappropriately discharged on AST without a valid indication.

\section{Conclusions}

This audit identified an important area for improvement in GICU patient care. AST is known to increase risk of nosocomial infection, rebound acid secretion, drug side effects and unnecessary costs. ${ }^{4}$ The audit intervention led to a notable improvement, and hence reduction in the attributed risks. Ongoing education of staff is recommended to establish longterm change.

\section{Conflict of interest statement}

No conflicting interests declared.

\section{References}

1 Sesler JM. Stress-related mucosal disease in the intensive care unit: an update on prophylaxis. AACN Adv Crit Care 2007;18:119-28.

2 St George's GICU. An introduction to the general intensive care unit (CHAOS) handbook. Feb 2016: p54.

3 NICE. Gastro-oesophageal reflux disease and dyspepsia in adults: investigation and management. NICE guidelines CG184. September 2014. www.nice.org.uk/Guidance/CG184

4 Howell MD, Novack V, Grgurich P et al. Iatrogenic gastric acid suppression and the risk of nosocomial Clostridium difficile infection. Arch Intern Med 2010;170:784-90.

Authors: ${ }^{1}$ Royal Surrey County Hospital, Guildford, UK; ${ }^{2}$ Worthing Hospital, Worthing, UK 